\title{
RECUPERAÇÃO DE METAIS DE CATALISADORES MÁSSICOS E MONOMETÁLICOS
}

\section{Júlio Carlos Afonso*, Tatiana Siqueira de Lima, Paula Constante Campos e Ana Acácia de Sá Pinheiro}

Departamento de Química Analítica, Instituto de Química, Universidade Federal do Rio de Janeiro, CP 68563, 21949-900 Rio de Janeiro - RJ

Recebido em 14/8/02; aceito em 11/3/03

\begin{abstract}
RECOVERY OF METALS FROM MONOMETALLIC AND NON-SUPPORTED CATALYSTS. This work presents a study on the dissolution of some commercial monometallic and non-supported deactivated catalysts in $\mathrm{HF}+\mathrm{H}_{2} \mathrm{O}_{2}$ mixtures (and, eventually, other media) under mild experimental conditions, after a previous oxidation step. The samples were neither crushed nor grinded. The best experimental conditions were dependent on the nature of the support and of the active phase. For example, the $\mathrm{Pt} / \mathrm{Al}_{2} \mathrm{O}_{3}$ catalyst was dissolved in about 10 minutes, without agitation and heating; however, dissolution of the $\mathrm{Pd} / \mathrm{Al}_{2} \mathrm{O}_{3}, \mathrm{Ni} / \mathrm{Al}_{2} \mathrm{O}_{3}, \mathrm{Ni} /$ $\mathrm{SiO}_{2}, \mathrm{Cu} / \mathrm{Al}_{2} \mathrm{O}_{3}$ and $\mathrm{V}_{2} \mathrm{O}_{5}$ samples required a temperature of $60{ }^{\circ} \mathrm{C}$ and an agitation of $400 \mathrm{rpm}$. A careful addition of a $\mathrm{NaOH}$ solution allowed a quantitative precipitation of aluminium as criolite $\left(\mathrm{Na}_{3} \mathrm{AlF}_{6}\right)$ or precipitation of $\mathrm{Si}$ as $\mathrm{Na}_{2} \mathrm{SiF}_{6} ; \mathrm{NaF}$ was obtained as a by-product. As expected, processing of $\mathrm{Pd} / \mathrm{C}, \mathrm{V}_{2} \mathrm{O}_{5}$ and $\mathrm{CuO} \cdot \mathrm{Cr}_{2} \mathrm{O}_{3}$ samples was relatively simple. Metals recovery from catalysts reached a quantitative level in all samples studied; it is particularly interesting that platinum and palladium could be easily recovered in a single step process, thus separing them from aluminium.
\end{abstract}

Keywords: spent catalyst; metals recovery; fluoride ion.

\section{INTRODUÇÃO}

Materiais contendo $\mathrm{Ni}, \mathrm{Cu}$ e metais preciosos - $\mathrm{Au}, \mathrm{Ag}$ e os metais platínicos (Ru, Os, Rh, Ir, Pd, Pt) - são largamente empregados na indústria na manufatura de componentes eletrônicos, em eletrodeposições, no preparo de catalisadores e na síntese de produtos químicos (química fina). Isso é devido às peculiares propriedades físicas e químicas dos compostos desses elementos. A fim de prevenir a poluição ambiental, preservar o meio ambiente e recuperar os metais, faz-se necessário desenvolver tecnologias adequadas de reciclagem dos mesmos, presentes nos materiais usados ${ }^{1}$. Por exemplo, estima-se que 15,5 toneladas de metais do grupo da Pt estarão disponíveis a cada ano a partir de conversores catalíticos usados, somente na América do Norte ${ }^{2}$. Da mesma forma, a reciclagem desses mesmos metais presentes em catalisadores de reforma causará impacto na economia das indústrias de refino de petróleo ${ }^{3}$.

A reciclagem de materiais contendo metais nobres é facilmente justificada por motivos econômicos. Com efeito, os fornecedores de catalisadores à base desses metais costumam vendê-los com a condição de retorno da amostra usada, para recuperação do elemento presente. Mais exatamente, muito embora o teor de metais nobres em um catalisador comercial esteja na faixa de $1 \%(\mathrm{p} / \mathrm{p})$ ou menos, eles correspondem a mais de $60 \%$ do custo total desse produto ${ }^{4}$.

A literatura indica uma profunda mudança no perfil qualitativo e quantitativo das publicações efetuadas na área de reciclagem de catalisadores industriais usados, conforme se vê na Tabela 1 . O número de artigos e revisões publicados em literatura aberta aumentou significativamente, em detrimento da quantidade de patentes apresentadas. Este panorama justifica-se pelo interesse crescente da comunidade científica internacional e industrial quanto a problemas ambientais, no qual está inserida a questão do destino a ser dado aos catalisadores desativados (estes contêm componentes tóxicos - compostos cancerígenos e metais pesados - que são sérios poluentes de solos, plantas e lençóis freáticos).

*e-mail: julio@iq.ufrj.br
Tabela 1. Panorama geral das publicações em reciclagem de catalisadores usados

\begin{tabular}{lrr}
\hline Período & $1993-2001$ & $1975-1992$ \\
\hline Patentes & $48(38,7 \%)$ & $66(75,0 \%)$ \\
Revisões & $18(14,5 \%)$ & $4(4,5 \%)$ \\
Artigos & $58(46,8 \%)$ & $18(20,5 \%)$ \\
\hline TOTAL & $124(100 \%)$ & $88(100 \%)$ \\
\hline
\end{tabular}

Os processos de reciclagem propostos na literatura, apesar da grande variedade de métodos, mostram uma clara evolução ao longo das décadas. Inicialmente, como os catalisadores desativados eram considerados como "minérios" ou "fontes minerais" dos elementos de interesse, foram adaptados processos piro- ou hidrometalúrgicos tal como aplicados às amostras minerais naturais. Esses métodos envolvem o uso de drásticas condições experimentais (calcinações, fusões etc) e, conseqüentemente, implicam em elevado consumo energético.

Atualmente, as pesquisas estão dirigidas para o desenvolvimento de processos conduzidos em condições experimentais brandas. Em muitos casos procede-se a uma etapa prévia, que pode ser uma redução ${ }^{5-9}$ ou uma queima controlada do coque ${ }^{5-6}$. Nesses casos, procura-se simplificar a composição da amostra e/ou reduzir certos elementos da fase ativa (como os metais nobres) ao estado elementar, enquanto que outros componentes do catalisador (especialmente o suporte) são dissolvidos em meio apropriado ( $\mathrm{NaOH}$ ou ácidos não oxidantes). Outras rotas prevêem a dissolução total da amostra em meio ácido oxidante ${ }^{10-11}$. Como o suporte corresponde à maior parte em peso do catalisador, o consumo de reagente é considerável, e gera-se muito resíduo. No caso da dissolução total, o uso de misturas água régia ou sulfo-nítrica é largamente predominante. Todavia, se o processo for conduzido a mais de $70{ }^{\circ} \mathrm{C}$, torna-se difícil na prática industrial devido à alta corrosividade do meio, à grande geração de gases tóxicos $\left(\mathrm{HCl}, \mathrm{Cl}_{2}, \mathrm{NO}_{\mathrm{x}}\right.$, etc) e às perdas frequientes dos próprios reagentes. A inclusão de novos suportes ampliou a necessidade 
de desenvolver processos compatíveis para esses materiais. Por exemplo, no caso do suporte ser carvão, este pode ser eliminado (juntamente com o coque) após cuidadosa oxidação prévia, e dissolução da cinza em meio adequado. É o caso dos estudos efetuados com o catalisador $\mathrm{Pd} / \mathrm{C}^{5,9,12,13}$. Menos discutido é o processamento de catalisadores mássicos (sem suporte); embora menos empregados, têm-se exemplos industriais bastante importantes, como os catalisadores à base de ferro na síntese de amônia, o pentóxido de vanádio na manufatura de ácido sulfúrico e a cromita de cobre $\left(\mathrm{CuO} . \mathrm{Cr}_{2} \mathrm{O}_{3}\right)$, empregada em hidrogenações, combustão, decomposição de álcoois, reforma de hidrocarbonetos não aromáticos, conversão do monóxido de carbono com água ou óxidos de nitrogênio, alquilação e oxidação do etanol a acetaldeído ${ }^{14}$. É importante mencionar que no desenvolvimento de novos catalisadores, muitas vezes os estudos se iniciam com catalisadores mássicos, exatamente para melhor avaliar a fase ativa, evitando efeitos decorrentes da influência do suporte sobre a dispersão e a atividade dessa fase ativa. Do mesmo modo, pode-se inferir que estes materiais podem servir para um estudo didático sobre o processamento de lixo tecnológico com vistas a recuperar os metais presentes em suas formulações, pois a composição é basicamente mais simples que a de catalisadores suportados: somente a química do(s) elemento(s) da fase ativa é relevante nesse processamento.

A análise da literatura mostra que é extremamente escassa a menção ao uso do íon fluoreto, ao contrário dos demais haletos (cloreto, brometo e iodeto), no processamento de catalisadores desativados ${ }^{15}$. No momento, não se tem uma clara razão para esse fato, muito embora se possa suspeitar que seja devido ao caráter corrosivo e poluente do fluoreto. Por outro lado, esse íon é uma base bem mais dura que os demais íons haleto e, como tal, forma complexos muito estáveis com cátions com configuração de gases nobres (os chamados ácidos duros). Isso ocorre, em especial, nos íons com carga elevada e raio iônico pequeno, e um exemplo ${ }^{16}$ é o íon $\mathrm{Al}^{3+}$, presente no composto $\mathrm{Al}_{2} \mathrm{O}_{3}$, freqüentemente empregado como suporte de catalisadores. Outro aspecto a ser destacado é que muitos catalisadores contêm $\mathrm{SiO}_{2}$ como suporte ou aditivo ao óxido $\mathrm{Al}_{2} \mathrm{O}_{3}$. O dióxido de silício é um composto bastante refratário, mas é solúvel em soluções ácidas contendo fluoreto (formação de ácido hexafluorossilícico - $\mathrm{H}_{2} \mathrm{SiF}_{6}$ ), sob condições brandas.

Em função do exposto acima, este trabalho objetivou empregar o íon fluoreto para a dissolução de catalisadores comerciais mássicos e monometálicos suportados, aproveitando as propriedades complexantes que ele possui. Foi dada ênfase ao uso de condições brandas de trabalho, conforme a tendência observada na literatura nos últimos anos. Este trabalho procurou contribuir para a formação de uma literatura aberta sobre um assunto de grande interesse estratégico e tecnológico.

\section{PARTE EXPERIMENTAL}

\section{Catalisadores}

Empregaram-se catalisadores monometálicos comerciais $\mathrm{X} / \mathrm{Al}_{2} \mathrm{O}_{3}$ ( $\mathrm{X}=\mathrm{Pd}, \mathrm{Pt}, \mathrm{Ni}$ e $\mathrm{Cu}$ ), Ni/SiO$, \mathrm{V}_{2} \mathrm{O}_{5},\left(99,5 \%\right.$ p/p), $\mathrm{Pd} / \mathrm{C}$ e CuO.Cr $\mathrm{C}_{2} \mathrm{O}_{3}$ ( $25 \% \mathrm{p} / \mathrm{p}$ de $\mathrm{Cu}$ e $47 \% \mathrm{p} / \mathrm{p}$ de $\mathrm{Cr}$ ), mantidos em suas formas originais (esferas de diâmetro 1-2 mm). Os teores típicos (\% p/p) dos catalisadores suportados virgens e desativados estão apresentados na Tabela 2. O catalisador à base de platina foi empregado em uma unidade de reforma catalítica de hidrocarbonetos em refinaria de petróleo, por cerca de 3 meses. Os catalisadores de paládio foram utilizados em hidrogenações de compostos de química fina por cerca de 1 ano; os catalisadores à base de níquel foram usados em plantas de hidrogenação de óleos vegetais durante cerca de 1 ano; o catalisador à base de cobre foi empregado em análises de combustão por 6 meses; a amostra de $\mathrm{V}_{2} \mathrm{O}_{5}$ proveio de unidade de síntese de ácido sulfúrico pelo método de contato, enquanto que o catalisador $\mathrm{CuO} . \mathrm{Cr}_{2} \mathrm{O}_{3}$ proveio de hidrogenações em química fina. Nenhuma dessas amostras foi regenerada durante suas vidas úteis.

\section{Tratamento prévio das amostras}

As amostras foram submetidas a um tratamento prévio de oxidação ao ar em mufla $\left(500{ }^{\circ} \mathrm{C}, 1 \mathrm{~atm}, 5 \mathrm{~h}\right.$, sem fluxo de ar). A finalidade deste pré-tratamento foi: a) eliminar o coque eventualmente presente (como $\mathrm{CO} / \mathrm{CO}_{2}$ ) e a água (catalisadores $\mathrm{X} / \mathrm{Al}_{2} \mathrm{O}_{3}, \mathrm{Ni} / \mathrm{SiO}_{2}$ ); b) eliminar a água, o coque e o suporte no caso do catalisador $\mathrm{Pd} / \mathrm{C}$ e c) eliminar a umidade dos catalisadores mássicos $\mathrm{V}_{2} \mathrm{O}_{5}$ e $\mathrm{CuO} \cdot \mathrm{Cr}_{2} \mathrm{O}_{3}$. Apesar de não terem teor significativo de carbono, o tratamento dessas amostras visou também colocar todas elas, de naturezas e aplicações industriais distintas, em um mesmo pré-tratamento antes da etapa de processamento propriamente dito.

\section{Processos de dissolução}

Basicamente, foram usados dois reagentes: ácido fluorídrico $37 \%$ $(\mathrm{p} / \mathrm{p})\left(\approx 20 \mathrm{~mol} \mathrm{~L}^{-1}\right)$ e peróxido de hidrogênio a $36 \%(\mathrm{p} / \mathrm{p})$. A massa

Tabela 2. Dados de análise química* dos catalisadores

\begin{tabular}{lcccc}
\hline Catalisador desativado & Metal & $\mathrm{Al}$ ou Si & $\mathrm{Fe}$ & $\mathrm{C}$ \\
\hline $\mathrm{Pt} / \mathrm{Al}_{2} \mathrm{O}_{3}$ & $0,39 \pm 0,04$ & $50,6 \pm 0,3$ & $0,05 \pm 0,01$ & $3,5 \pm 0,3$ \\
$\mathrm{Pd} / \mathrm{Al}_{2} \mathrm{O}_{3}$ & $0,48 \pm 0,02$ & $50,9 \pm 0,5$ & $0,15 \pm 0,03$ & $3,0 \pm 0,2$ \\
$\mathrm{Ni} / \mathrm{Al}_{2} \mathrm{O}_{3}$ & $4,97 \pm 0,04$ & $47,7 \pm 0,3$ & $0,05 \pm 0,01$ & $2,0 \pm 0,1$ \\
$\mathrm{Cu} / \mathrm{Al}_{2} \mathrm{O}_{3}$ & $4,83 \pm 0,04$ & $47,7 \pm 0,5$ & $0,10 \pm 0,01$ & $2,2 \pm 0,2$ \\
$\mathrm{Ni} / \mathrm{SiO}_{2}$ & $4,74 \pm 0,02$ & $48,1 \pm 0,5$ & $0,10 \pm 0,02$ & $1,8 \pm 0,2$ \\
$\mathrm{Pd} / \mathrm{C}$ & $0,50 \pm 0,03$ & - & $0,05 \pm 0,01$ & $\mathrm{C}$ \\
\hline Catalisador virgem & Metal & $\mathrm{Al}$ ou Si & Fe & - \\
\hline $\mathrm{Pt} / \mathrm{Al}_{2} \mathrm{O}_{3}$ & $0,39 \pm 0,02$ & $52,8 \pm 0,4$ & - \\
$\mathrm{Pd} / \mathrm{Al}_{2} \mathrm{O}_{3}$ & $0,50 \pm 0,03$ & $53,2 \pm 0,6$ & $0,02 \pm 0,01$ & - \\
$\mathrm{Ni} / \mathrm{Al}_{2} \mathrm{O}_{3}$ & $5,01 \pm 0,05$ & $48,8 \pm 0,3$ & Não detectado & - \\
$\mathrm{Cu} / \mathrm{Al}_{2} \mathrm{O}_{3}$ & $5,04 \pm 0,06$ & $48,6 \pm 0,5$ & $0,03 \pm 0,01$ & - \\
$\mathrm{Ni} / \mathrm{SiO}_{2}$ & $5,02 \pm 0,04$ & $48,9 \pm 0,5$ & Não detectado & Não detectado \\
$\mathrm{Pd} / \mathrm{C}$ & $0,51 \pm 0,02$ & - & $99,5 \pm 0,2$ \\
\hline
\end{tabular}

* obtidos por absorção atômica, exceto o carbono (combustor Leco); valores expressos em \% (p/p) com intervalo de confiança a 95\% 
de catalisador, em geral, foi fixada em 1,0 g. A partir dos dados de análise quantitativa dos catalisadores (Tabela 2), a massa de íon fluoreto foi calculada de modo que ele complexasse todos os metais presentes no catalisador, com um excesso de $10 \%$ (p/p) em relação às reações teóricas de formação de fluorocomplexos. Os experimentos foram conduzidos com os reagentes sozinhos ou com misturas dos mesmos em diversas proporções volumétricas (razões $\mathrm{HF} / \mathrm{H}_{2} \mathrm{O}_{2}$ de 0 até 6 ). Os experimentos foram feitos em béquer de teflon sobre placa agitadora-aquecedora, sob agitação (0 - $400 \mathrm{rpm})$, a uma temperatura variando entre 25 e $70{ }^{\circ} \mathrm{C}$, pelo período necessário à dissolução total da amostra ou à obtenção de uma massa insolúvel permanente. A reprodutibilidade dos experimentos foi confirmada através da execução dos mesmos em duplicata (catalisadores $\mathrm{V}_{2} \mathrm{O}_{5} \mathrm{e}$ $\mathrm{CuO} . \mathrm{Cr}_{2} \mathrm{O}_{3}$ ) ou triplicata (demais casos). A solução obtida foi diretamente empregada na etapa de fracionamento dos elementos presentes na mesma.

\section{Métodos analíticos}

Os metais foram dosados por absorção atômica (Perkin Elmer AAS 3300), cujos limites de detecção, determinados experimentalmente, são: Al: 0,5 mg L-1 ; Cr e Cu: $0,05 \mathrm{mg} \mathrm{L}^{-1}$; Ni e Pd: $0,1 \mathrm{mg} \mathrm{L}^{-1}$; Pt, Si e V: $1 \mathrm{mg} \mathrm{L}^{-1}$. O teor de carbono foi determinado via análise elementar (Combustor LECO). As dosagens quantitativas foram complementadas por análises qualitativas ${ }^{17}$ para as soluções e os sólidos. Os íons $\mathrm{Na}^{+}$e $\mathrm{F}^{-}$foram dosados através da cromatografia de íons (Dionex DX 100, limite de detecção 0,01 $\mathrm{mg} \mathrm{L}^{-1}$ ). Foi, ainda utilizada a difração de raios-X para a análise de diversos sólidos obtidos na etapa de isolamento dos elementos após a dissolução dos catalisadores (difratômetro PHILIPS PW 1820; fonte de radiação $\mathrm{Cu}-\mathrm{K} \alpha$, varredura angular (2 teta) $12-70^{\circ}$, com passo de $0,020^{\circ} \mathrm{e}$ tempo de $1 \mathrm{~s}$ por passo; as amostras apresentavam granulometria menor que $0,0044 \mathrm{~nm}$ ).

\section{RESULTADOS E DISCUSSÃO}

\section{Efeito da oxidação prévia}

A perda de massa decorrente da eliminação de voláteis situou-se na faixa $6-8 \%(\mathrm{p} / \mathrm{p})$ nos catalisadores $\mathrm{X} / \mathrm{Al}_{2} \mathrm{O}_{3}$ e Ni/SiO 2 estudados, percentual esse de $99,5 \%(\mathrm{p} / \mathrm{p})$ para a amostra de $\mathrm{Pd} / \mathrm{C}$ e de $2 \%(\mathrm{p} / \mathrm{p})$ para os catalisadores $\mathrm{V}_{2} \mathrm{O}_{5}$ e $\mathrm{CuO} \cdot \mathrm{Cr}_{2} \mathrm{O}_{3}$. A eliminação do carbono foi quantitativa; cerca de $85-90 \%$ (p/p) do ferro foi eliminado como fuligem de $\mathrm{Fe}_{2} \mathrm{O}_{3}$, e não foram observadas perdas dos demais metais por arraste ou volatilização durante o processo de oxidação.

\section{Determinação da mistura binária ideal de dissolução para o catalisador $\mathrm{Pt} / \mathrm{Al}_{2} \mathrm{O}_{3}$}

A Figura 1 mostra o perfil de dissolução do componente principal do catalisador, o suporte (alumina), no caso da amostra de Pt/ $\mathrm{Al}_{2} \mathrm{O}_{3}$. Razões $\mathrm{HF} / \mathrm{H}_{2} \mathrm{O}_{2}$ abaixo de 1 (isto é, excesso de água oxigenada) levaram a sérias perdas de fluoreto como HF por arraste no momento da dissolução, o que acarretou numa dissolução não quantitativa do suporte. A faixa ótima situou-se, para uma relação $\mathrm{HF} /$ $\mathrm{H}_{2} \mathrm{O}_{2}$, entre 1 e 5. Contudo, o uso de excesso crescente de HF levou a tempos cada vez maiores para se obter a dissolução total do suporte, inclusive com perda da solubilização quantitativa caso a razão fosse maior do que 5 .

A reação de dissolução do catalisador $\mathrm{Pt} / \mathrm{Al}_{2} \mathrm{O}_{3}$ foi altamente exotérmica, a temperatura passou rapidamente de 25 para cerca de $75{ }^{\circ} \mathrm{C}$, diminuindo gradualmente após cerca de $3 \mathrm{~min}$. Tal fato está associado à decomposição da água oxigenada catalisada pela plati- na. A solubilização quantitativa do suporte deu-se em aproximadamente $10 \mathrm{~min}$.

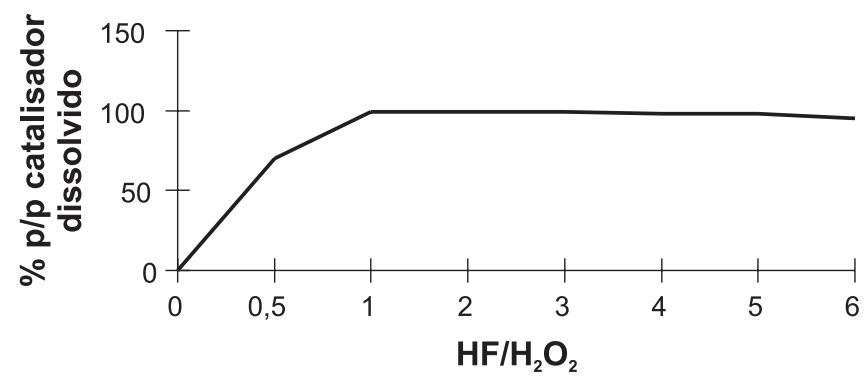

Figura 1. Dissolução do catalisador Pt/alumina em função da razão HF/ $\mathrm{H}_{2} \mathrm{O}_{2}(v / v)$

\section{Tempo de dissolução}

Tendo como parâmetro a solubilização quantitativa do suporte, o tempo de dissolução do catalisador Pt/alumina foi de apenas $10 \mathrm{~min}$ para misturas $\mathrm{HF} / \mathrm{H}_{2} \mathrm{O}_{2}$ na faixa de 1:1 até 4:1.

\section{Estabilidade das soluções}

A estabilidade mostrou-se um fator crítico, principalmente quando um processamento imediato da solução não era efetuado. Razões acima de 2:1 levaram a uma rápida instabilidade da mesma, com deposição de um precipitado branco cuja composição exata não foi determinada.

Dos resultados experimentais, pode-se avaliar que a melhor mistura de dissolução tem a razão volumétrica 1:1- o tempo de dissolução é minimo, obtém-se uma boa estabilidade da solução final frente ao tempo, e evita-se um excesso desnecessário de fluoreto, um ponto de grande importância pois o fluoreto é um sério poluente do meio ambiente.

Após a solubilização da amostra, a adição de $\mathrm{HCl}$ ou $\mathrm{HNO}_{3}$ aos extratos possibilitou o aumento da estabilidade para acima de 60 dias. Isso não terá, em geral, interesse prático, pois o que se faz comumente em matéria de recuperação de metais é o processamento imediato da solução obtida após a dissolução do catalisador.

\section{Dissolução dos catalisadores $\mathrm{Ni}, \mathrm{Cu}$ e $\mathrm{Pd} / \mathrm{Al}_{2} \mathrm{O}_{3}$}

Com base nos resultados para o catalisador $\mathrm{Pt} / \mathrm{Al}_{2} \mathrm{O}_{3}$, tentou-se dissolver os catalisadores acima nas mesmas condições otimizadas (mistura $\mathrm{HF} / \mathrm{H}_{2} \mathrm{O}_{2}$ 1:1, sem agitação e aquecimento induzidos). Contudo, os resultados foram totalmente diferentes daqueles observados no caso do catalisador à base de platina: a dissolução do suporte foi muito reduzida, da ordem de $10 \%$ p/p, e a decomposição do peróxido de hidrogênio foi extremamente lenta, não acarretando em efeitos térmicos ou físicos sobre a mistura reacional. Desse modo, fez-se um estudo de otimização da dissolução desses catalisadores com a mistura $\mathrm{HF} / \mathrm{H}_{2} \mathrm{O}_{2} 1: 1$, por meio da variação da temperatura da mistura reacional e da agitação. O critério de análise foi, mais uma vez, a solubilização do suporte. A chave da dissolução dos catalisadores monometálicos é a reação de complexação do alumínio, segundo a Equação 1:

$\mathrm{Al}_{2} \mathrm{O}_{3}+12 \mathrm{HF} \longleftrightarrow 2\left[\mathrm{AlF}_{6}\right]^{3-}+3 \mathrm{H}_{2} \mathrm{O}+6 \mathrm{H}^{+}$

Os resultados experimentais (Tabelas 3 e 4) indicam que a dissolução do suporte desses catalisadores era conseguida caso se empre- 
gasse temperatura da ordem de $60^{\circ} \mathrm{C}$ e agitação da ordem de $400 \mathrm{rpm}$. Nessas condições, a dissolução completou-se em aproximadamente 20 min. A decomposição do peróxido de hidrogênio manteve-se em ritmo suave, mostrando-se essencial ao processo de abertura. O uso de HF puro levava a uma solubilização quantitativa somente após $3 \mathrm{~h}$ de aquecimento. Fatores de ordem física (geração de calor e turbulência no meio reacional devido à liberação de $\mathrm{O}_{2}$ ), que auxiliam no processo de solubilização das amostras, podem explicar o efeito positivo do $\mathrm{H}_{2} \mathrm{O}_{2}$ na abertura dos catalisadores em estudo.

Tabela 3. Otimização do processo de dissolução dos catalisadores* em $\mathrm{HF}+\mathrm{H}_{2} \mathrm{O}_{2} 1: 1$ com agitação de $400 \mathrm{rpm}$

\begin{tabular}{ccc}
\hline $\begin{array}{c}\text { Temperatura } \\
\left({ }^{\circ} \mathrm{C}\right)\end{array}$ & $\begin{array}{c}\%(\mathrm{p} / \mathrm{p}) \text { alumina } \\
\text { dissolvida }\end{array}$ & $\begin{array}{c}\text { Tempo de } \\
\text { dissolução }(\mathrm{min})\end{array}$ \\
\hline 25 & $13 \pm 3$ & $70 \pm 6$ \\
35 & $37 \pm 3$ & $48 \pm 5$ \\
40 & $62 \pm 4$ & $36 \pm 5$ \\
50 & $90 \pm 4$ & $30 \pm 3$ \\
60 ou 70 & $99 \pm 2$ & $20 \pm 2$ (dissolução total) \\
\hline
\end{tabular}

* $\mathrm{X} / \mathrm{Al}_{2} \mathrm{O}_{3}(\mathrm{X}=\mathrm{Ni}, \mathrm{Cu}$ e $\mathrm{Pd})$; ** dosagem de $\mathrm{Al}$ por absorção atômica; intervalo de confiança a $95 \%$

Tabela 4. Otimização do processo de dissolução dos catalisadores* em $\mathrm{HF}+\mathrm{H}_{2} \mathrm{O}_{2}(1: 1)$ à temperatura de $60{ }^{\circ} \mathrm{C}$

\begin{tabular}{ccc}
\hline $\begin{array}{c}\text { Agitação } \\
(\mathrm{rpm})\end{array}$ & $\begin{array}{c}\%(\mathrm{p} / \mathrm{p}) \text { alumina } \\
\text { dissolvida** }\end{array}$ & $\begin{array}{c}\text { Tempo de } \\
\text { dissolução (min) }\end{array}$ \\
\hline 50 & $30 \pm 4$ & $48 \pm 5$ \\
100 & $53 \pm 6$ & $45 \pm 5$ \\
200 & $84 \pm 5$ & $35 \pm 3$ \\
250 & $90 \pm 5$ & $28 \pm 2$ \\
300 & $95 \pm 5$ & $25 \pm 2$ \\
400 ou 450 & $100 \pm 2$ & $20 \pm 1$ (dissolução total) \\
\hline
\end{tabular}

* $\mathrm{X} / \mathrm{Al}_{2} \mathrm{O}_{3}(\mathrm{X}=\mathrm{Ni}, \mathrm{Cu}$ e $\mathrm{Pd})$; ** dosagem de $\mathrm{Al}$ por absorção atômica; intervalo de confiança a $95 \%$

\section{Comparação entre $\mathrm{Pt} / \mathrm{Al}_{2} \mathrm{O}_{3}$ e os demais catalisadores monometálicos suportados em alumina}

A fim de compreender os diferentes resultados experimentais obtidos em termos de dissolução destes catalisadores, a Tabela 5 resume os principais dados de caracterização físico-química das amostras quanto ao suporte e à fase metálica existente.

Os dados desta tabela indicam que o efeito da queima do coque é diferente segundo o metal considerado e que parte do coque redu- ziu a platina ao estado elementar, aparentemente com um baixo grau de reoxidação. A platina reduzida, finamente dispersa sobre o suporte ("negro de platina"), catalisa a decomposição do peróxido de hidrogênio. Assim, após um período de indução (cerca de 5 min), iniciou-se a decomposição vigorosa do $\mathrm{H}_{2} \mathrm{O}_{2}$, o que liberou calor, ao mesmo tempo em que a liberação de grande quantidade de oxigênio gasoso em curto período de tempo provocava uma forte turbulência no meio reacional. Por outro lado, as características da alumina do catalisador $\mathrm{Pt} / \mathrm{Al}_{2} \mathrm{O}_{3}$ favorecem amplamente a dissolução em tempo curto, devido à elevada área específica associada a um considerável volume de poros. Este fato facilita o ataque do $\mathrm{HF}$ ao suporte $\mathrm{Al}_{2} \mathrm{O}_{3}$, acelerando a formação do fluorocomplexo $\mathrm{AlF}_{6}{ }^{3-}$.

Com relação aos outros catalisadores os metais estão essencialmente na forma de óxidos, que são muito menos efetivos na decomposição do peróxido de hidrogênio que os metais. Mesmo que haja redução dos óxidos pelo coque, a reoxidação dos metais parece ser quantitativa. De fato, frente ao oxigênio, o $\mathrm{Pd}$, o $\mathrm{Ni}$ e o $\mathrm{Cu}$ são mais facilmente oxidáveis do que a Pt. O PdO é insolúvel em $\mathrm{HF}^{18}$, o que cria uma barreira à dissolução rápida do $\mathrm{Al}_{2} \mathrm{O}_{3} \cdot \mathrm{CuO}$ e $\mathrm{NiO}$ solubilizam-se nesse ácido:

$\mathrm{XO}+4 \mathrm{HF} \longleftrightarrow\left[\mathrm{XF}_{4}\right]^{2-}+\mathrm{H}_{2} \mathrm{O}+2 \mathrm{H}^{+}(\mathrm{X}=\mathrm{Cu}, \mathrm{Ni})$

mas o processo só é perceptível sob aquecimento, o que também retardou a dissolução do suporte. Além disso, o suporte dos catalisadores correspondentes é menos poroso e possui área específica bem menor que no caso do $\mathrm{Pt} / \mathrm{Al}_{2} \mathrm{O}_{3}$. Tudo isso justifica a necessidade de se empregar aquecimento e agitação para obter a dissolução quantitativa do suporte.

\section{Recuperação dos metais}

Os dados de análise quantitativa do Pd e da Pt estão consignados na Tabela 6 , e confirmam as expectativas da literatura ${ }^{18}$, indicando que tanto a Pt como o PdO são insolúveis em HF, ao contrário dos óxidos de $\mathrm{Ni}, \mathrm{Cu}$ e $\mathrm{Pt}\left(\mathrm{PtO}_{2}\right)$. Esta propriedade reveste-se de grande interesse prático, pois permite uma recuperação quantitativa e imediata do metal nobre, separando-o facilmente da grande quantidade de alumínio presente. Em geral, esta recuperação excede os níveis que são normalmente observados na literatura ${ }^{4-11}$ (valores que vão desde 60 até $97 \%(\mathrm{p} / \mathrm{p})$ ), independente de que os processos empregados sejam piro/hidrometalúrgicos ou em condições mais brandas.

\section{Processamento do catalisador $\mathbf{P d} / \mathrm{C}$}

Os estudos de recuperação do metal nobre (Pd), contido na amostra $\mathrm{Pd} /$ carvão, foram bastante simples. Após a eliminação do coque e do suporte por queima controlada a $600{ }^{\circ} \mathrm{C}$ por $5 \mathrm{~h}$ (sem fluxo de ar), a cinza de $\mathrm{PdO}$, de cor marrom escura, tinha alta pureza e foi obtida

Tabela 5. Caracterização físico-química dos catalisadores monometálicos suportados em $\mathrm{Al}_{2} \mathrm{O}_{3}$

\begin{tabular}{|c|c|c|c|c|}
\hline Parâmetro & $\mathrm{Pt} / \mathrm{Al}_{2} \mathrm{O}_{3}$ & $\mathrm{Pd} / \mathrm{Al}_{2} \mathrm{O}_{3}$ & $\mathrm{Cu} / \mathrm{Al}_{2} \mathrm{O}_{3}$ & $\mathrm{Ni} / \mathrm{Al}_{2} \mathrm{O}_{3}$ \\
\hline $\begin{array}{l}\text { Estado da fase metálica } \\
\text { após a queima do coque }\end{array}$ & $\begin{array}{c}\mathrm{Pt}^{\circ}->99 \%(\mathrm{p} / \mathrm{p}) \\
\mathrm{PtO}_{2}-\text { balanço }\end{array}$ & $\mathrm{PdO}=100 \%(\mathrm{p} / \mathrm{p})$ & $\mathrm{CuO}=100 \%(\mathrm{p} / \mathrm{p})$ & $\mathrm{NiO}=100 \%(\mathrm{p} / \mathrm{p})$ \\
\hline $\begin{array}{l}\text { Caracterização físico-química } \\
\text { da alumina do catalisador }\end{array}$ & $\begin{array}{c}\mathrm{S}_{\mathrm{BET}}: 200 \mathrm{~m}^{2} / \mathrm{g} \\
\text { Volume de poro: } \\
0,5 \mathrm{~mL} / \mathrm{g}\end{array}$ & $\begin{array}{c}\mathrm{S}_{\mathrm{BET}}: 100 \mathrm{~m}^{2} / \mathrm{g} \\
\text { Volume de poro: } \\
0,2 \mathrm{~mL} / \mathrm{g}\end{array}$ & $\begin{array}{c}\mathrm{S}_{\mathrm{BET}}: 90 \mathrm{~m}^{2} / \mathrm{g} \\
\text { Volume de poro: } \\
0,2 \mathrm{~mL} / \mathrm{g}\end{array}$ & $\begin{array}{c}\mathrm{S}_{\mathrm{BET}}: 120 \mathrm{~m}^{2} / \mathrm{g} \\
\text { Volume de poro: } \\
0,4 \mathrm{~mL} / \mathrm{g}\end{array}$ \\
\hline
\end{tabular}

* área específica BET e volume de poros determinados por meio de adsorção de $\mathrm{N}_{2}$ a $-196^{\circ} \mathrm{C}$. As isotermas de adsorção foram obtidas em um equipamento Micromeritics ASAP 2010. As amostras foram submetidas a um pré-tratamento, sob vácuo, a $300{ }^{\circ} \mathrm{C}$ por $1 \mathrm{~h}$. 
Tabela 6. Análise de metais* após a dissolução do suporte em $\mathrm{HF}+\mathrm{H}_{2} \mathrm{O}_{2} 1: 1$

\begin{tabular}{llll}
\hline Catalisador & $\%(\mathrm{p} / \mathrm{p})$ precipitado & $\%(\mathrm{p} / \mathrm{p})$ solúvel & Observações \\
\hline Pt/alumina(1) & $99,7 \pm 0,2\left(\mathrm{Pt}^{0}\right)$ & $0,3 \pm 0,1$ & Solução praticamente incolor, tom esverdeado $\left(\right.$ traços de $\left.\mathrm{PtF}_{6}{ }^{2-}\right)$ \\
$\mathrm{Pd} /$ alumina(2) & $100,0 \pm 0,2(\mathrm{PdO})$ & Desprezível & Solução incolor $\left(\right.$ apenas $\left.\mathrm{AlF}_{6}{ }^{3-}\right)$ \\
Ni/alumina(2) & Desprezível & $100,0 \pm 0,2\left(\mathrm{Ni}^{2+}\right)$ & Solução verde $\left(\mathrm{AlF}_{6}{ }^{3-}+\mathrm{NiF}_{4}{ }^{2-}\right)$ \\
$\mathrm{Cu} /$ alumina(2) & Desprezível & $100,0 \pm 0,2\left(\mathrm{Cu}^{2+}\right)$ & Solução azul $\left(\mathrm{AlF}_{6}{ }^{3-}+\mathrm{CuF}_{4}{ }^{2-}\right)$ \\
\hline
\end{tabular}

* obtida por absorção atômica; intervalo de confiança a 95\%; 1 . sem agitação e à temperatura ambiente; 2 . processo a $60{ }^{\circ} \mathrm{C}$ e $400 \mathrm{rpm}$

com rendimento quantitativo $(>98 \%$ (p/p) em relação à massa teórica de $\mathrm{PdO}$ ). Este rendimento é compatível com os estudos já mostrados na literatura ${ }^{5,9,12,13}$. Contudo, o óxido foi de difícil solubilização em ácidos, mesmo concentrados e aquecidos (talvez devido à redução drástica da área superficial). O principal cuidado aqui foi evitar um espalhamento das cinzas de óxido de paládio pelo interior da mufla, o que levaria a consideráveis perdas de rendimento. Com o objetivo de minimizar essas perdas, tampou-se frouxamente o recipiente contendo o catalisador com um vidro de relógio em pyrex, deixando a amostra ao abrigo de correntes convectivas dentro da mufla.

\section{Processamento da solução contendo $\mathrm{Al}\left(\mathrm{AlF}_{6}{ }^{3-}\right)$}

A Figura 2 mostra o fluxograma simplificado utilizado no isolamento dos compostos $\mathrm{Na}_{3} \mathrm{AlF}_{6}$ e NaF. A solução (ácida) foi paulatinamente neutralizada com solução $6 \mathrm{~mol} \mathrm{~L}^{-1} \mathrm{de} \mathrm{NaOH}$, gota a gota

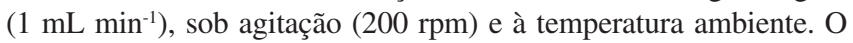
pH final deve ficar entre 6 e 7, conforme a amostra tratada (ver adiante). A criolita, $\mathrm{Na}_{3} \mathrm{AlF}_{6}$, precipitou como um sólido branco extremamente fino (solubilidade em água de $419 \mathrm{mg} \mathrm{L}^{-1}$ ), sendo filtrado e lavado 2-3 vezes com água (contendo $10 \mathrm{mg} \mathrm{L}^{-1} \mathrm{de} \mathrm{HF}$ ) a $60{ }^{\circ} \mathrm{C}$ ( $4 \mathrm{~mL} \mathrm{~g}^{-1}$ precipitado). A seguir, foi seco em estufa a $150{ }^{\circ} \mathrm{C}$ por $1 \mathrm{~h}$. $\mathrm{O}$ difratograma de raios-X é inteiramente concordante com o da criolita padrão. Com base no teor de alumínio dos catalisadores (Tabela 2), a criolita contém mais de $95 \%$ (p/p) do Al presente, e as análises químicas demonstraram que o grau de pureza excede a $99,5 \%$ $(\mathrm{p} / \mathrm{p})$ (menos de $0,1 \%(\mathrm{p} / \mathrm{p})$ dos metais da fase ativa), o que torna este material utilizável na metalurgia do alumínio. No caso dos catalisadores $\mathrm{Cu} / \mathrm{Al}_{2} \mathrm{O}_{3}$ e Ni/ $\mathrm{Al}_{2} \mathrm{O}_{3}$, os metais da fase ativa achavamse solubilizados. Por isso, a neutralização com $\mathrm{NaOH}$ foi levada até um pH levemente ácido (cerca de 6, ao invés de 7 para as demais amostras) para evitar a co-precipitação do níquel ou do cobre com a criolita. Uma vez isolada a criolita, mais $\mathrm{NaOH}$ foi adicionado, nas mesmas condições experimentais quando da precipitação do alumínio, para elevar o $\mathrm{pH}$ a cerca de 9; os hidróxidos de níquel ou de cobre precipitaram. Eles foram filtrados e lavados com água até resultado negativo para fluoreto e sódio no líquido de lavagem (tipicamente, $8 \mathrm{~mL} \mathrm{~g}^{-1}$ precipitado). $\mathrm{O}$ efluente mais as águas de lavagem foram reunidos e tratados com $\mathrm{HF}$ até $\mathrm{pH}$ entre 7 e 8 (formação de $\mathrm{NaF}$ adicional); a seguir, esta solução foi evaporada. O difratograma de raios- $\mathrm{X}$ era equivalente ao do $\mathrm{NaF}$ padrão; de fato, ele continha mais de $99,5 \%$ (p/p) desse sal (análise por cromatografia de íons). A solução final após o isolamento da criolita dos catalisadores $\mathrm{Pt} / \mathrm{Al}_{2} \mathrm{O}_{3}$ e $\mathrm{Pd} / \mathrm{Al}_{2} \mathrm{O}_{3}$ continha essencialmente $\mathrm{NaF}$ e algum $\mathrm{HF}$ não neutralizado. A evaporação da mesma forneceu um sólido branco cristalino cuja análise por cromatografia de íons mostrou que ele também era composto por mais de $99,5 \%$ (p/p) de NaF, tendo aplicação metalúrgica.

Todos os resíduos finais (evaporados) contêm tipicamente menos de $10 \mathrm{ppm}$ de $\mathrm{Al}^{3+}$ e menos de $0,1 \%$ (p/p) do total dos elementos da fase ativa. O processamento de catalisadores monometálicos suportados em alumina empregando fluoreto não gerou resíduos finais em quantidade apreciável.

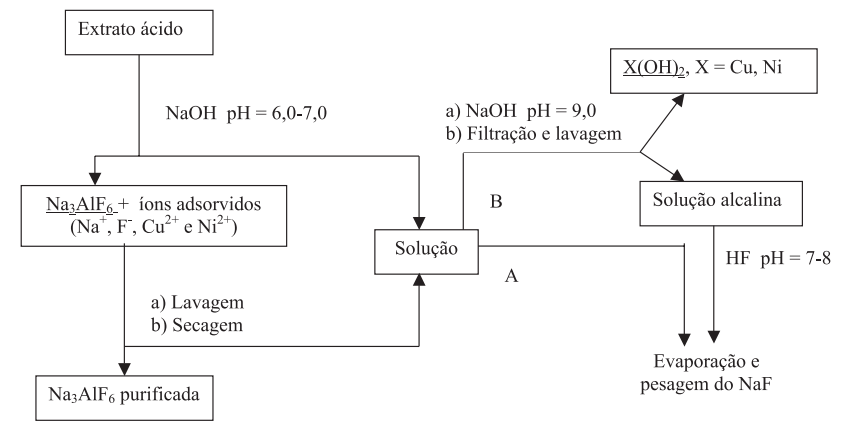

Figura 2. Fluxograma para o isolamento da criolita e do fluoreto de sódio ( $\mathrm{A}$ - catalisadores Pt e $\mathrm{PdAl}_{2} \mathrm{O}_{3} ; \mathrm{B}$ - catalisadores $\mathrm{Cu}$ e $\mathrm{Ni} / \mathrm{Al}_{2} \mathrm{O}_{3}$ )

O fluoreto presente nos dois produtos finais $\left(\mathrm{Na}_{3} \mathrm{AlF}_{6} \mathrm{e} \mathrm{NaF}\right)$ equivale a cerca de $95 \% \mathrm{p} / \mathrm{p}$ de todo o fluoreto presente na mistura de dissolução. Este dado serve para justificar bem a necessidade de se usar algum excesso de HF em relação ao teórico, para compensar perdas durante a dissolução do catalisador (cerca da metade do HF em excesso perdeu-se sob nossas condições experimentais). Como o íon fluoreto é um sério poluente do meio ambiente, medidas se fazem necessárias para que os $5 \%$ p/p deste elemento perdidos (basicamente no momento da abertura das amostras) sejam absorvidos por um meio apropriado (água, por exemplo) para fins de proteção ambiental. O que se fez neste trabalho foi a passagem do efluente oriundo da etapa de abertura em água destilada, onde a concentração de HF foi ajustada a $10 \mathrm{mg} \mathrm{L}^{-1}$ para ser usada na lavagem da criolita obtida ou, então, empregada na neutralização do efluente após isolamento do níquel ou do cobre.

\section{Processamento do catalisador $\mathrm{Ni} / \mathrm{SiO}_{2}$}

A faixa ótima de dissolução quantitativa foi a mistura $\mathrm{HF} / \mathrm{H}_{2} \mathrm{O}_{2}$ de 2:1 a 5:1; um excesso de $\mathrm{H}_{2} \mathrm{O}_{2}$ levava a uma forte perda de $\mathrm{F}^{-}$ (como HF), o que acarretava numa dissolução parcial do catalisador; por outro lado, um excesso de HF conduzia a uma dissolução extremamente lenta, com perda de rendimento da dissolução acima de 5:1. A reação exigiu aquecimento, sendo a faixa ótima entre $60-70{ }^{\circ} \mathrm{C}$, ou seja, o mesmo intervalo utilizado para os catalisadores anteriormente estudados, suportados em alumina (exceto o $\mathrm{Pt} / \mathrm{Al}_{2} \mathrm{O}_{3}$ ). A solução é verde devido ao complexo $\mathrm{NiF}_{4}{ }^{2-}$. A dissolução completouse em $1 \mathrm{~h}$, mais lenta que nos catalisadores à base de alumina (cerca de 20 min), devido à cinética de reação do $\mathrm{SiO}_{2}$ com o $\mathrm{HF}$ ser mais lenta do que com o $\mathrm{Al}_{2} \mathrm{O}_{3}$. O desempenho inferior da mistura $\mathrm{HF}+$ $\mathrm{H}_{2} \mathrm{O}_{2} 1: 1$, considerada a melhor para a solubilização dos catalisadores suportados em alumina, deve estar ligado à cinética de reação. A dissolução do catalisador obedeceu às reações:

$$
\begin{aligned}
& \mathrm{SiO}_{2}+6 \mathrm{HF} \longleftrightarrow \mathrm{SiF}_{6}{ }^{2-}+2 \mathrm{H}^{+}+2 \mathrm{H}_{2} \mathrm{O} \\
& \mathrm{NiO}+4 \mathrm{HF} \longleftrightarrow\left[\mathrm{NiF}_{4}\right]^{2-}+\mathrm{H}_{2} \mathrm{O}+2 \mathrm{H}^{+}
\end{aligned}
$$


A primeira etapa do fracionamento foi o isolamento da maior parte do silício pela adição de solução $6 \mathrm{~mol} \mathrm{~L}^{-1}$ de $\mathrm{NaOH}$, gota a gota $\left(1 \mathrm{~mL} \mathrm{~min}^{-1}\right)$, sob agitação $(200 \mathrm{rpm})$ e à temperatura ambiente. O precipitado (branco) foi lavado com água a $10-15{ }^{\circ} \mathrm{C}\left(4 \mathrm{~mL} \mathrm{~g}^{-1}\right.$ sólido) para a remoção do Ni. A seguir, ele foi seco em estufa a $150{ }^{\circ} \mathrm{C}$ por $1 \mathrm{~h}$, resultando numa massa branca, contendo tipicamente $70 \%(\mathrm{p} / \mathrm{p})$ do silício presente no suporte. Os dados de difração de raios-X confirmam a formação de $\mathrm{Na}_{2} \mathrm{SiF}_{6}$; a análise por cromatografia de íons indicou um grau de pureza superior a 99,5\% (p/p).

À solução verde foi adicionado $\mathrm{NaOH}\left(6 \mathrm{~mol} \mathrm{~L}^{-1}\right)$ até ajustar o $\mathrm{pH}$ em 9, precipitando um composto verde-maçã, correspondendo ao $\mathrm{Ni}(\mathrm{OH})_{2}$. O efluente líquido final era totalmente incolor. As análises de Ni por absorção atômica (a partir da dissolução do óxido em ácido nítrico $3 \mathrm{~mol} \mathrm{~L}^{-1}$ ) mostraram uma recuperação de praticamente $100 \%$ (p/p), fato esse não observado quando se empregam outros ácidos (clorídrico, sulfúrico $)^{10-11}$, por conta da insolubilidade do silicato de níquel nesses meios (ao contrário do que acontece em HF).

Após o isolamento do $\mathrm{Ni}$, ajustou-se o $\mathrm{pH}$ da solução na faixa de 7-8 com HF (6 mol L-1), evaporando-se em seguida. Obteve-se um sólido branco fosco, cuja difração de raios-X acusou tratar-se de uma mistura de $\mathrm{Na}_{2} \mathrm{SiF}_{6}$ e NaF. Os dados de cromatografia de íons indicam que a composição típica desse sólido é $55 \%(\mathrm{p} / \mathrm{p}) \mathrm{de}_{2} \mathrm{NiF}_{6} \mathrm{e}$ $45 \%$ (p/p) de $\mathrm{NaF}$, concentrando $30 \%(\mathrm{p} / \mathrm{p})$ do silício original do suporte. Ele não pode ser descartado no ambiente, pois o fluoreto é um sério poluente ambiental. $\mathrm{O}$ sólido não pode ir in natura para aterros industriais, pois é um material lixiviável pela chuva, com o tempo. Para resolver este problema, pode-se tratar este sólido com $\mathrm{Ca}(\mathrm{OH})_{2} / \mathrm{Na}_{2} \mathrm{CO}_{3}$ para o acondicionamento do mesmo em aterro industrial (classe I). Porém, o NaF tem também um uso siderúrgico, como lubrificante no lingotamento do ferro. Nesse caso, chega-se à conclusão de que o processamento de catalisadores suportados em sílica em meio contendo fluoreto é capaz de não gerar resíduos químicos.

\section{Processamento do catalisador $\mathrm{V}_{2} \mathrm{O}_{5}$}

$\mathrm{O}$ processamento do catalisador $\mathrm{V}_{2} \mathrm{O}_{5}$ não mostrou dificuldades práticas, e o emprego de HF sozinho levou a uma solubilização do catalisador em cerca de $1 \mathrm{~h}$, sob agitação (400 rpm) e aquecimento da ordem de $60{ }^{\circ} \mathrm{C}$. Obteve-se uma solução amarela, contendo cátions $\mathrm{VO}_{2}^{+}$:

$\mathrm{V}_{2} \mathrm{O}_{5}+2 \mathrm{HF} \longleftrightarrow 2 \mathrm{VO}_{2}^{+}+\mathrm{H}_{2} \mathrm{O}+2 \mathrm{~F}^{-}$

A adição de peróxido de hidrogênio à solução de HF não alterou significativamente a cinética de solubilização do catalisador. Ao se tratar a solução com hidróxido de amônio $\left(6 \mathrm{~mol} \mathrm{~L}^{-1}\right)$ até $\mathrm{pH}$ 7, o sal vanadato de amônio, $\mathrm{NH}_{4} \mathrm{VO}_{3}$, de cor branca, precipitou:

$\mathrm{VO}_{2}^{+}+2 \mathrm{NH}_{4} \mathrm{OH}+\mathrm{F}^{-} \longleftrightarrow \mathrm{NH}_{4} \mathrm{VO}_{3}+\mathrm{NH}_{4} \mathrm{~F}+\mathrm{H}_{2} \mathrm{O}$

$\mathrm{O}$ filtrado, incolor, continha $\mathrm{NH}_{4} \mathrm{~F}$ (fluoreto de amônio). Esta solução pode ser tratada com sais de $\mathrm{Ca}^{2+}$ (tipicamente cloreto de cálcio) para precipitação do fluoreto como $\mathrm{CaF}_{2}$, obtendo-se uma solução de $\mathrm{NH}_{4} \mathrm{Cl}\left(\sim 5,0 \mathrm{mg} \mathrm{L}^{-1}\right)$, que pode ser descartada em águas de classe 8 (para navegação comercial e tratamento paisagístico), segundo a legislação brasileira ${ }^{19}$.

O sal de vanádio acima pode ser calcinado a $300{ }^{\circ} \mathrm{C}$ por 3-4 h, obtendo-se novamente o óxido de vanádio $(\mathrm{V})$ com grau de pureza acima de $99,5 \%(\mathrm{p} / \mathrm{p})$ :

$2 \mathrm{NH}_{4} \mathrm{VO}_{3} \longrightarrow \mathrm{V}_{2} \mathrm{O}_{5}+2 \mathrm{NH}_{3}+\mathrm{H}_{2} \mathrm{O}$ o efluente gasoso continha $\mathrm{NH}_{3}$, que pode ser facilmente absorvido em água fria ou em solução ácida $(\mathrm{HCl}$, etc). Tanto a gravimetria quanto as análises por absorção atômica indicam uma recuperação de praticamente $100 \%$ (p/p) do vanádio. O uso de ácido clorídrico no lugar do fluorídrico revelou-se ineficaz; a solubilização foi lenta, mesmo a quente, e ocorreu uma reação redox, com o vanádio sendo reduzido ao número de oxidação +4 e liberação de gás cloro.

\section{Processamento do catalisador $\mathrm{CuO}_{2} \mathrm{Cr}_{2} \mathrm{O}_{3}$}

O HF não conseguiu dissolver quantitativamente o cobre, mas o $\mathrm{HCl}$ conseguiu dissolvê-lo a quente $\left(60{ }^{\circ} \mathrm{C}\right)$, após 30-40 min sob agitação (400 rpm). O óxido $\mathrm{Cr}_{2} \mathrm{O}_{3}$, altamente refratário, é insolúvel tanto em $\mathrm{HF}$ como em $\mathrm{HCl}^{16-18}$. As análises da solução obtida por absorção atômica confirmaram as expectativas acima mencionadas: nela só havia cobre, correspondendo em média a $97 \%(\mathrm{p} / \mathrm{p})$ de todo o elemento presente no catalisador quando se empregou o $\mathrm{HCl}$ :

$\mathrm{CuO}+4 \mathrm{HCl} \longleftrightarrow\left[\mathrm{CuCl}_{4}\right]^{2-}+\mathrm{H}_{2} \mathrm{O}+2 \mathrm{H}^{+}$

Este resultado é muito interessante, constitui-se num caso onde uma extração seletiva de componentes do catalisador teve sucesso: obteve-se uma solução ácida contendo cobre e um resíduo final verde escuro, correspondendo ao $\mathrm{Cr}_{2} \mathrm{O}_{3}$. A evaporação da solução forneceu cristais verdes de $\mathrm{CuCl}_{2} .4 \mathrm{H}_{2} \mathrm{O}$. Alternativamente, tratou-se esta solução com $\mathrm{NaOH}\left(6 \mathrm{~mol} \mathrm{~L}^{-1}\right)$ até $\mathrm{pH} 7$, onde precipitou o $\mathrm{Cu}(\mathrm{OH})_{2}$, tendo como resíduo final uma solução de $\mathrm{NaCl}$, contendo menos de 1 ppm de $\mathrm{Cu}^{2+}$.

\section{CONCLUSÕES}

A combinação de uma etapa de oxidação controlada do catalisador e o emprego de um meio $\mathrm{HF} / \mathrm{H}_{2} \mathrm{O}_{2}$ ajustado permitiram obter uma dissolução quantitativa dos suportes alumina e sílica em tempo bastante curto. $\mathrm{O}$ íon fluoreto permitiu a separação direta e quantitativa do metal nobre, no momento da dissolução da amostra, sob condições experimentais brandas, pelo menos quando o suporte é a alumina. As condições ótimas para a dissolução desta última parecem depender de suas características físico-químicas e do estado da fase metálica após a queima do coque.

Foi possível obter produtos finais contendo fluoreto com alto grau de pureza, com aplicações industriais, e que concentram cerca de $95 \% \mathrm{p} / \mathrm{p}$ de todo o fluoreto presente na mistura reacional de dissolução.

Para a seqüência desta atividade de pesquisa, está sendo estudado o processamento de catalisadores contendo simultaneamente alumínio e silício (como é o caso das zeólitas), e também o comportamento de uma mesma fase ativa depositada em suportes diferentes, a fim de precisar melhor o efeito da natureza do suporte e de sua interação com a fase ativa na recuperação dos componentes do catalisador.

\section{AGRADECIMENTOS}

T. S. de Lima, P. C. Campos e A. A. de S. Pinheiro agradecem ao PBIC CNPq/SR-2-UFRJ a concessão de bolsas de iniciação científica.

Ao CNPq, CAPES, FUJB (Fundação Universitária José Bonifácio), CENPES e à Fundação de Amparo à Pesquisa do Estado do Rio de Janeiro (FAPERJ), pelo auxílio financeiro. Ao Laboratório de Desenvolvimento Analítico (LADA/DQA/IQ/UFRJ) pelas análises por cromatografia de íons. 


\section{REFERÊNCIAS}

1. Nakahiro, Y.; Trans. Mater. Soc. Jpn. 1994, 18 A (Ecomaterials), 167.

2. Mishra, R. K.; Precious Metals 1993, 17, 449.

3. Rosso, J. P.; Precious Metals 1992, 16, 361.

4. Mhaske, A. A.; Dhadke, P. M.; Appl. Catal., A 2001, 212, 153.

5. Han, K. N.; Meng, X.; US pat. 5.542 .957 1996. (CA 125: P147553r)

6. Golebrowski, A.; Gorecka, B.; Janecki, Z.; Buzon, A.; Hryniewicz, I.; Janiczek, W.; Chemik 1994, 47, 392.

7. Mhaske, A. A.; Dhadke, P. M.; Hydrometallurgy 2001, 61, 143.

8. Wu, K. Y. A.; Wisecaryer, K. D.; Abraham, M. A.; Takach, N.; Yang, N.; Precious Metals 1993, 17, 343

9. Herszkiewicz, A.; Jaworska, D.; Patente Polonesa 164.796 1994. (CA 125: P334785e)

10. Angelidis, T. N.; Skouraki, E.; Appl. Catal., A 1996, 142, 387.

11. Rabah, A.; Metal Powder Report 1998, 53, 37.
12. Sun, D. D.; Tay, J. H.; Cheong, H. K.; Leung, D. L. K.; Qian, G. R.; J. Hazard. Mater. 2001, 87, 213.

13. Brezovicky, J.; Dolezci, P.; Koren, M.; Fotyi, D.; Kovacs, L.; Gala, M.; Halomi, M.; Dudek, I.; Patente Tcheca 276.107 1992. (CA 121: P62179r)

14. Jebarathinam, N. J.; Bull. Chem. Soc. Jpn. 1994, 67, 3334.

15. Lima, T. S.; Campos, P. C.; Afonso, J. C.; Boletim Técnico da Petrobrás 1998, 41, 169.

16. Vogel, A. I.; Análise Quantitativa Inorgânica, $4^{\mathrm{a}}$ ed, Guanabara Dois: São Paulo, 1983, p. 195-196.

17. Feigl, F.; Spot Tests in Inorganic Analysis, Elsevier: Amsterdam, 1958, cap. 3.

18. Lurie, L.; Handbook of Analytical Chemistry, Mir: Moscou, 1978, cap. 6 e 10.

19. Resolução No 20 do Conselho Nacional do Meio Ambiente (CONAMA), de 18/06/1986, publicada no Diário Oficial da União em 30/07/1986. 